\title{
Effect of Magnesium Sulfate Combined with Phentolamine and Nifedipine for Gestational Hypertension and Serum Levels of LIF and Apelin
}

\author{
Jie Wen ${ }^{1}$ and Xiaojing Li ${ }^{2}$
}

\begin{abstract}
Objective: To investigate the effect of magnesium sulfate combined with phentolamine and nifedipine for the treatment of gestational hypertension and on the levels of serum LIF and Apelin.

Study Design: An experimental study.

Place and Duration of Study: Obstetrics and Gynecology Clinics, The Affiliated Hospital North China University of Science and Technology, China, from September 2016 to February 2018.

Methodology: One hundred and sixty patients with gestational hypertension were randomly divided into a control group and an observation group, 80 patients in each group. Control group was given magnesium sulfate alone, while observation group was added with phentolamine and nifedipine on the basis of the treatment in control group. Curative effects, pregnancy outcomes, and levels of serum LIF and Apelin were compared.

Results: The total effective rate of treatment in the observation group was higher than that in the control group $(p=0.005)$. After treatment, level of serum LIF in the observation group was higher than that in the control group $(p<0.001)$, and level of serum Apelin in the observation group was lower than that in the control group $(p<0.001)$. Incidence of premature birth, cesarean section and neonatal asphyxia in the observation group were all lower than those in the control group $(p=0.005$, $p<0.001$ and $p=0.005$, respectively), while there was no significant difference in the incidence of neonatal death between the two groups $(p=0.316)$.

Conclusion: Magnesium sulfate combined with phentolamine and nifedipine has a better therapeutic effect on gestational hypertension, which can effectively regulate the levels of serum LIF and Apelin and improve pregnancy outcomes.
\end{abstract}

Key Words: Gestational hypertension, Magnesium sulfate, Phentolamine, Nifedipine, LIF, Apelin.

\section{INTRODUCTION}

Gestational hypertension is a common complication of pregnancy. It can cause miscarriage, placental abruption, premature birth and massive bleeding in pregnant women, which will pose a serious threat to the health and safety of pregnant women and fetuses.1,2 The etiology and pathogenesis of gestational hypertension are not fully understood, and placental or trophoblastic ischemia and hypoxia is one of the many etiological theories. The theory holds that the declined trophoblastic infiltration during pregnancy in the placental formation stage in patients with gestational hypertension leads to vascular remodeling disorder, reduced placental perfusion, ischemia and hypoxia of trophoblasts, the release of large amounts of cytokines

\footnotetext{
1 Department of Obstetrics and Gynecology, The Affiliated Hospital North China University of Science and Technology, HeBei Province, 063000, China

2 Department of Gynecology, The Second Affiliated Hospital of Medical College of Zhejiang University, 310000, China

Correspondence: Xiaojing Li, Department of Gynecology, The Second Affiliated Hospital of Medical College of Zhejiang University, 310000, China

E-mail:zik963@163.com

Received: June 19, 2018; Accepted: September 28, 2018
}

and eventually the occurrence of gestational hypertension. ${ }^{3}$

Studies have shown that leukemia inhibitory factor (LIF) is a multifunctional cytokine distributed in the uterine decidua and placental cells, which can affect the infiltration ability of trophoblast cells, and is related to the occurrence and development of gestational hypertension and perinatal prognosis to some extent. ${ }^{4}$ Apelin is a small molecule active peptide newly discovered in recent years. It is collectively expressed in placental tissues of placental vascular endothelial cells, syncytiotrophoblasts, cytotrophoblasts, and villous interstitium. ${ }^{5}$ Related studies have shown that Apelin has special physiological and pathological implications in the homeostasis of the placental vascular system during pregnancy. 6

The basic purpose of treatment for gestational hypertension is to effectively prevent and control eclampsia, thereby reducing its perinatal prevalence and mortality and improving the prognosis of mother and child.7,8 However, conventional antihypertensive and antispasmodic drugs have unsatisfactory results with different degrees of adverse reactions. Therefore, exploring safe and effective therapeutic drugs for gestational hypertension is of great value to improve the safety and health of pregnant women. 
Table I: Comparison of the clinical efficacy between the two groups.

\begin{tabular}{|c|c|c|c|c|c|c|c|}
\hline Group & $\mathrm{n}$ & Significant & Effective & Improved & Invalid & Total effective & p-value \\
\hline Control group & 80 & $30(37.50 \%)$ & $28(35.00 \%)$ & $8(10.00 \%)$ & $14(17.50 \%)$ & $66(82.50 \%)$ & 0.005 \\
\hline Observation group & 80 & $45(56.25 \%)$ & $26(32.50 \%)$ & $6(7.50 \%)$ & $3(3.75 \%)$ & $77(96.25 \%)$ & \\
\hline
\end{tabular}

The purpose of this study was to investigate the curative effect of magnesium sulfate combined with phentolamine and nifedipine in the treatment of gestational hypertension and analyze its effect on the levels of serum LIF and Apelin, in order to provide reference for clinical treatment of gestational hypertension.

\section{METHODOLOGY}

This study was conducted at Obstetrics and Gynecology Clinics, The Affiliated Hospital North China University of Science and Technology, China, from September 2016 to February 2018. A total of 160 patients with gestational hypertension were selected as subjects. Inclusion criteria were patients diagnosed as gestational hypertension by blood pressure test, in combination with their clinical manifestations and signs, patients with treatment indications for magnesium sulfate combined with phentolamine and nifedipine; and patients and their families who understood the research content and treatment risk and were willing to cooperate. Exclusion criteria were patients with essential hypertension and a history of hypertension before pregnancy; and patients with severe comorbid conditions such as function abnormalities in liver, kidney, heart and lung, or malignant tumors. The patients were randomly divided into a control group and an observation group, with 80 patients in each group.

Both groups received routine symptomatic and supportive treatment, including oxygen uptake, sedation, and diuresis. The control group was given magnesium sulfate

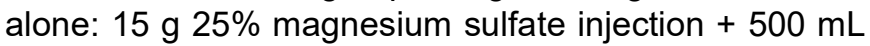
$5 \%$ glucose injection through intravenous infusion at a speed of $2 \mathrm{~g} / \mathrm{h}$. Another vein channel was opened in the observation group based on the treatment of the control group: 20 mg phentolamine injection $+250 \mathrm{~mL} 5 \%$ glucose injection through intravenous infusion at a speed of 20 $\mathrm{mg} / \mathrm{h}$; and meanwhile, $30 \mathrm{mg}$ nifedipine tablets was combined at 1 time/d sublingually. The two groups were treated continuously for 7 days.

The clinical efficacy of the two groups was compared. Significant: proteinuria and edema disappeared, and the mean arterial pressure index recovered. Effective: proteinuria and edema symptoms significantly improved, and the mean arterial pressure decreased by $20 \mathrm{mmHg}$ or more. Improved: proteinuria and edema symptoms improved, and the mean arterial pressure decreased by $10 \mathrm{mmHg}$ or more. Invalid: proteinuria and edema symptoms did not change or increased, and the mean arterial pressure decreased by $10 \mathrm{mmHg}$ or less. Total effective rate $=$ (significant + effective + improved cases $) /$ total number of cases $\times 100 \%$. The morning fasting
Table II: Comparison of the levels of serum LIF and apelin before and after treatment between the two groups.

\begin{tabular}{l|l|l|c|c}
\hline Index & Time & $\begin{array}{c}\text { Control } \\
\text { group } \\
(\mathrm{n}=80)\end{array}$ & $\begin{array}{c}\text { Observation } \\
\text { group } \\
(\mathrm{n}=80)\end{array}$ & p-value \\
\hline LIF (ng/L) & Before treatment & $676.94 \pm 38.24$ & $675.51 \pm 28.64$ & 0.789 \\
& After treatment & $706.30 \pm 47.79$ & $763.06 \pm 27.03$ & $<0.001$ \\
\hline Apelin (ng/L) & Before treatment & $376.32 \pm 23.87$ & $377.15 \pm 33.41$ & 0.857 \\
& After treatment & $263.86 \pm 22.05$ & $214.78 \pm 25.55$ & $<0.001$ \\
\hline
\end{tabular}

Table III: Comparison of the pregnancy outcomes between the two groups.

\begin{tabular}{l|c|c|r}
\hline Pregnancy outcomes & $\begin{array}{c}\text { Control group } \\
(\mathrm{n}=80)\end{array}$ & $\begin{array}{c}\text { Observation group } \\
(\mathrm{n}=80)\end{array}$ & P-value \\
\hline Premature birth & $12(15.00 \%)$ & $2(2.50 \%)$ & 0.005 \\
Cesarean section & $49(61.25 \%)$ & $26(32.50 \%)$ & $<0.001$ \\
Neonatal asphyxia & $12(15.00 \%)$ & $2(2.50 \%)$ & 0.005 \\
Neonatal death & $1(1.25 \%)$ & $0(0)$ & 0.316 \\
\hline
\end{tabular}

cubital venous blood ( $3 \mathrm{~mL}$ ) was collected from all patients before and after treatment. The levels of serum LIF and Apelin were detected by enzyme-linked immunosorbent assay (ELISA). The pregnancy outcomes of the two groups were compared.

SPSS 23.0 software was used for statistical analysis. Measurement data are expressed in mean $\pm S D$ and independent sample t-test was used. Count data was expressed in $\mathrm{n}(\%)$ and Chi-square test was used. $\mathrm{P}<0.05$ indicates statistically significant difference.

\section{RESULTS}

Among the 160 patients, the age ranged from 20 to 34 years, with an average of $28.75 \pm 2.36$ years; the gestational age ranged from 28 to 39 weeks, with an average of $36.61 \pm 3.43$ weeks; the systolic pressure was $140.84-180.57 \mathrm{mmHg}$, with an average of $143.86 \pm 12.52$ $\mathrm{mmHg}(1 \mathrm{mmHg}=0.133 \mathrm{kPa})$; and the diastolic pressure $78.55-102.46 \mathrm{mmHg}$, with an average of $95.74 \pm 9.38$ $\mathrm{mmHg}$.

The total effective rate of treatment in the observation group was $96.25 \%$ (77 cases), which was significantly higher than that of the control group $82.50 \%$ (66 cases) $(p=0.005)$, as shown in Table I.

Before treatment, there was no significant difference in levels of serum LIF and Apelin between the two groups $(p=0.789$ and $p=0.857$, respectively). After treatment, the level of serum LIF in the observation group was higher than that in the control group $(p<0.001)$, and the level of serum Apelin in the observation group was lower than that in the control group $(p<0.001)$, as shown in Table II. 
The premature birth rate in the observation group was $2.50 \%$ ( 2 cases), which was lower than $15.00 \%$ (12 cases) of the control group $(p=0.005)$; the incidence of cesarean section in the observation group was $32.50 \%$, which was lower than $61.25 \%$ (49 cases) of the control group $(p<0.001)$; the incidence of neonatal asphyxia in the observation group was $2.50 \%$ (2 cases), which was lower than $15.00 \%$ (12 cases) of the control group $(p=0.005) ;$ and there was no significant difference in the incidence of neonatal death between the two groups $(p=0.316)$, as shown in Table III.

\section{DISCUSSION}

Effective control of blood pressure in patients with gestational hypertension is the key to ensure maternal and child safety as well as delivery quality. Magnesium sulfate is a commonly used antihypertensive agent in patients with pregnancy-induced hypertension syndrome. It is safer. After intravenous administration, it regulates the neuromuscular transmission mechanism by inhibiting peripheral nerve and muscle and the release of acetylcholine in peripheral vessels, relaxes the blood vessels as well as the bones, relieves the vasospasm, and improves the state of organ hypoxia and ischemia. ${ }^{9}$ However, studies have found that the effect of pure magnesium sulfate is too short to guarantee a stable blood pressure in patients. ${ }^{10}$

Phentolamine is a novel alpha-blocker that reduces peripheral resistance by expanding smooth muscle of pulmonary artery and bronchi, and also has a reflex reinforcement effect on myocardial contractility to increase cardiac output.11 At the same time, phentolamine can effectively improve renal blood flow by regulating the renal artery, and the efficacy of diuresis and detumescence is significant. 12

Nifedipine is a calcium channel blocker drug that can relax systemic blood vessels, especially in the relief of coronary artery spasm. It is able to keep blood pressure low and blood concentration for as long as 24 hours, so its antihypertensive effect is stable and long-lasting. 13,14 This study found that the total effective rate of clinical treatment in the observation group was higher than that of the control group, which is consistent with the result of Xiao-Mei et al. 15 In terms of pregnancy outcomes, the incidences of cesarean section, neonatal asphyxia and premature delivery in the observation group were all lower than those of the control group, indicating that magnesium sulfate combined with phentolamine and nifedipine can ensure the quality of delivery and the health of newborns.

LIF, as a leukemia inhibitory factor, has a multi-directional regulatory effect on differentiation, proliferation, and infiltration of gestational trophoblast cells. ${ }^{16}$ Insufficient secretion of LIF can cause incomplete trophoblastic invasion into the uterus spiral arterioles, and insufficient blood supply for the placenta. The hypoxic-ischemic state can stimulate the body to secrete related hormones with feedback, causing systemic arterial spasm and vascular endothelium damage, and hence the occurrence of pregnancy-induced hypertension. ${ }^{17}$

Apelin has two-way effects on blood pressure regulation in pregnant women: it not only promotes the synthesis of NO through the activation of nitric oxide synthase and exerts effective vasodilatory effects, but also has an effect of increasing blood pressure by directly acting on APJ receptors in vascular smooth muscle cells.18,19 For patients with gestational hypertension, the overexpression of Apelin may further increase blood pressure and aggravate the condition. 20

This study showed that after treatment, the level of serum LIF in the observation group was higher than that in the control group, and the level of serum Apelin in the observation group was lower than that in the control group, revealing that magnesium sulfate combined with phentolamine and nifedipine can more effectively regulate the levels of serum LIF and Apelin, and thus relieve hypertensive conditions in patients with gestational hypertension. The efficacy evaluation results showed that the curative effect of the observation group was significantly better than that of the control group, which is consistent with the more effective regulation of the levels of serum LIF and Apelin in the observation group.

\section{CONCLUSION}

Magnesium sulfate combined with phentolamine and nifedipine had a better therapeutic effect on gestational hypertension, which can effectively regulate the levels of serum LIF and Apelin and improve pregnancy outcomes. Therefore, it is suitable for treating gestational hypertension.

\section{REFERENCES}

1. Khaskheli MN, Baloch S, Sheeba A, Baloch S, Khan F. Labour induction with gestational hypertension: A great obstetric challenge. Pak J Med Sci 2017; 33:151-5.

2. Wang W, Fan D, Wang J, Wu S, Lu Y, He Y, et al. Association between hypertensive disorders complicating pregnancy and risk of placenta accreta: a meta-analysis and systematic review. Hypertens Pregnancy 2018; 7:168-74.

3. Hutten JWM, Kuijpers JC, Velzen DV, Wallenburg HCS. Pathogenesis of pregnancy-induced hypertensive disorders a review of experimental animal models. Clin Exp Hypertens $B$ 2009; 10:287-306.

4. Sawai K, Azuma C, Koyama M, Ito S, Hashimoto K, Kimura T, et al. Leukemia inhibitory factor (LIF) enhances trophoblast differentiation mediated by human chorionic gonadotropin (HCG). Biochem Biophys Res Commun 1995; 211:137-43.

5. Samareh Fekri M, Etminan A, Rashidnedjad A, Mojibian A, Masoomi Y. Serum apelin peptide level in hemodialysis patients with pulmonary arterial hypertension. Iran J Kidney Dis 2018; 12:209-14. 
6. Van MT, Doherty A, Baczyk D, Drewlo S, Baud D, Carvalho J, et al. Apelin in normal pregnancy and pregnancies complicated by placental insufficiency. Reprod Sci 2016; 23:1037-43.

7. Folk DM. Hypertensive disorders of pregnancy: Overview and current recommendations. J Midwifery Womens Health 2018; 63:289-300

8. Sutton ALM, Harper LM, Tita ATN. Hypertensive disorders in pregnancy. Obstet Gynecol Clin North Am 2018; 45:333-47.

9. Belfort MA, Saade GR, Moise KJ Jr. The effect of magnesium sulfate on maternal and fetal blood flow in pregnancy-induced hypertension. Acta Obstet et Gynecol Scand 2011; 72:526-30.

10. Bhatia RK, Bottoms SF, Sokol RJ. Pre-eclampsia, magnesium sulfate, and blood pressure evaluation during labor: A preliminary consideration. Am J Perinatol 1987; 4:352-5.

11. Prados-Frutos JC, Rojo R, González-Serrano J, GonzálezSerrano C, Sammartino G, Martínez-González JM, et al. Phentolamine mesylate to reverse oral soft-tissue local anesthesia: a systematic review and meta-analysis. J Am Dent Assoc 2015; 146:751-9.e3.

12. Cimmino VM, Bove EL, Argenta LC, Brown JW, Kirsh MM. The effect of simultaneous administration of levarterenol and phentolamine on renal blood flow. Ann Thorac Surg 1976; 21:158-63.

13. Songthamwat S, Na Nan C, Songthamwat M. Effectiveness of nifedipine in threatened preterm labor: a randomized trial. Int $J$ Womens Health 2018; 10:317-23.
14. Sridharan K, Sequeira RP. Drugs for treating severe hypertension in pregnancy: a network meta-analysis and trial sequential analysis of randomized clinical trials. $\mathrm{Br} J$ Clin Pharmacol 2018; 84:1906-16.

15. Xiaomei $Y$, Dingqing $G$. Clinical analysis of phentolamine magnesium sulfate combining with nifedipine in treatment of pregnancy induced hypertension. Mod Prev Med 2012; 23: 6137-8.

16. Suman P, Shembekar N, Gupta SK. Leukemia inhibitory factor increases the invasiveness of trophoblastic cells through integrated increase in the expression of adhesion molecules and pappalysin 1 with a concomitant decrease in the expression of tissue inhibitor of matrix metalloproteinases. Fertil Steril 2013; 99:533-42.

17. Jessmon $P$, Leach RE, Armant DR. Diverse functions of HBEGF during pregnancy. Mol Reprod Dev 2009; 76: 1116-27.

18. Gilbert JS. From apelin to exercise: emerging therapies for management of hypertension in pregnancy. Hypertens Res. 2017; 40:519-25.

19. Wang C, Liu X, Kong D, Qin X, Li Y, Teng X, et al. Apelin as a novel drug for treating preeclampsia. Exp Ther Med 2017; 14:5917-23.

20. Liao YM, Qiao FY. Expression of apelin in placentas of patients with hypertensive disorders complicating pregnancy. Zhonghua Fu Chan Ke Za Zhi 2007; 42:382-5. 